\title{
Online Community-based Design of Free and Open Source Software for Transgender Voice Training
}

\author{
ALEX A. AHMED, Northeastern University, Project Spectra, USA \\ BRYAN KOK, Project Spectra, USA \\ CORANNA HOWARD, Project Spectra, USA \\ KLEW STILL, Project Spectra, USA
}

\begin{abstract}
This paper describes Project Spectra, a collective of open source developers that aims to build free and open source voice training technology for transgender people. We demonstrate how a design prioritizing the agency of trans users was made possible through sustained community collaboration. Using an autoethnographic approach, we discuss our community-based design process, which was documented with memos, online meetings and text conversations, sketches, and other data sources. We illustrate how we articulated our values as a group: deciding our programming framework (including a Statement of Principles), elaborating our "Experience Goals" (the feelings we wanted our design to elicit), and determining the features we wanted to implement in our app. We conclude with a reflection on the benefits and challenges of conducting communitybased design research through an open-source organizational model.
\end{abstract}

CCS Concepts: • Human-centered computing $\rightarrow$ Participatory design; Collaborative content creation; • Social and professional topics $\rightarrow$ Gender.

Additional Key Words and Phrases: community-based collaborative design, free and open source software, transgender, feminist epistemologies, autoethnography

\section{ACM Reference Format:}

Alex A. Ahmed, Bryan Kok, Coranna Howard, and Klew Still. 2020. Online Community-based Design of Free and Open Source Software for Transgender Voice Training. Proc. ACM Hum.-Comput. Interact. 4, CSCW3, Article 258 (December 2020), 27 pages. https://doi.org/10.1145/3434167

\section{STATEMENT ON RESEARCHER POSITIONALITY}

Throughout this paper, first-person singular pronouns $(I, m e)$ are used to indicate the first author of this paper. First-person plural pronouns (we, our) refer to our collective broadly, and also specifically to those of us who volunteered to contribute to this paper (the authors). I cannot separate my own identity, concerns, and values from how the work was conducted. This work pays particular attention to how my own social location and academic position influenced the design process and its outcomes. Ultimately, we hope to paint a holistic picture of our organization and faithfully represent its members.

Authors' addresses: Alex A. Ahmed, ahmed.al@northeastern.edu, Northeastern University, Project Spectra, 360 Huntington Avenue, Boston, Massachusetts, 02115, USA; Bryan Kok, bryan.wyern1@gmail.com, Project Spectra, USA; Coranna Howard, me@komiga.com, Project Spectra, USA; Klew Still, klew1013@gmail.com, Project Spectra, USA.

Permission to make digital or hard copies of all or part of this work for personal or classroom use is granted without fee provided that copies are not made or distributed for profit or commercial advantage and that copies bear this notice and the full citation on the first page. Copyrights for components of this work owned by others than the author(s) must be honored Abstracting with credit is permitted. To copy otherwise, or republish, to post on servers or to redistribute to lists, requires prior specific permission and/or a fee. Request permissions from permissions@acm.org.

(c) 2020 Copyright held by the owner/author(s). Publication rights licensed to ACM.

2573-0142/2020/12-ART258 \$15.00

https://doi.org/10.1145/3434167

Proc. ACM Hum.-Comput. Interact., Vol. 4, No. CSCW3, Article 258. Publication date: December 2020. 


\section{INTRODUCTION}

Human-computer interaction (HCI) researchers are increasingly taking critical stances towards participatory design (PD) methods - such as workshops, focus groups, and "blue-sky" ideation exercises - arguing that they reinforce differences in power between researchers and participants $[40,42,57,62]$. Despite the ostensible benefits of opening design processes up to individual and community engagement, such attempts are fraught by histories of institutional racism [40], colonialist narratives of "development" [43], and the outsized influence of corporate power under capitalism, from which academia is not exempt $[33,41,46]$. These histories of oppression have marred attempts to design collaboratively, especially with marginalized people.

This paper builds on existing literature that critiques and reflects upon participatory and community-based collaborative design methods [6, 14, 40, 62]. For example, Harrington takes the "design workshop" as their object of analysis, citing examples of how the practice "misalign[s] with the lived experiences of underserved communities" [40]. Participants recounted histories of mistreatment from the institution with which the researchers were affiliated, and described the design activities as "childish." The authors argue that these tensions undermined PD's potential as a "democratic mechanism to respond to societal challenges," and that "reconstruction" is required to more closely align PD with community-based participatory research (CBPR), a methodology that aims for co-ownership and non-hierarchical, sustained collaboration between researchers and participants.

Whereas prior work focused on in-person collaborative methods, we will describe the possibilities and difficulties in facilitating a long-term community-based collaborative design project online. We ground this discussion in the context of designing and building a free and open source voice training app, with and for transgender people. We build on prior work exploring the voice- and technology-related values and challenges that trans people face [35, 36, 45, 63]. We argue that, given the widespread marginalization of trans people, it is essential for computing research to not only include us in design processes, but also to create sustainable structures for us to own and control the work from top to bottom: from sketching and brainstorming to prototyping, testing, and implementation.

We present Project Spectra, a collective dedicated to free and open source software (FOSS), which is a paradigm for the development of asynchronous, collaborative software development. FOSS primarily centers on code, not usability [32], and is not often used in HCI [24]. Despite a tendency towards openness, significant barriers preclude participation, particularly for women and people without technical skills [54]. We further existing research studying FOSS development cultures and practices from an HCI lens. Over the course of one and a half years, I (the first author) documented Project Spectra's design process - which included meeting notes, key decision points, and my own personal reflections - as a series of design memos. These memos, which contain sketches, code fragments, co-written documents, and screenshots from our app, serve as the primary data sources for this paper. I use autoethnography, a methodology that seeks to "describe and systematically analyze personal experience in order to understand cultural experience" [27]. Using my design memos as autoethnographic field notes, this paper explores the following questions:

- How did our organization work, and how was it structured?

- How did our design process shape our app, both functionally and aesthetically?

- How did my position as an academic researcher shape the design process?

In doing so, we hope to support researchers and practitioners in conducting community-based collaborative design projects through a case study focused on transgender voice. However, this paper does not - and ethically cannot - capture, summarize, or otherwise describe the beliefs, feelings and perspectives of each member of Project Spectra. Instead, I combine autoethnography

Proc. ACM Hum.-Comput. Interact., Vol. 4, No. CSCW3, Article 258. Publication date: December 2020. 
with a feminist epistemology, centering "subjectivity, emotionality, and the researcher's influence on research" [27]. Following Rosner and Haraway, this paper "stays with the trouble," focusing on moments of difficulty, contradictions, and breakdown [37, 62]. In summary, we contribute: (1) an application and reflection of an online collaborative design process; and (2) an overview of our effort's outcomes: the design goals, user testing, and implementation of a novel voice training app for trans people; (3) a set of recommendations for researchers and practitioners interested in community-based collaborative design.

\section{RELATED WORK}

This paper draws from, and builds on, two main lines of current thinking in CSCW: communitybased collaborative design, and understanding the conditions under which interactive systems both support and harm transgender people. Calling on the work of queer and trans scholars and activists, I describe how increasing public visibilty and acceptance of trans people is contingent on normative gender performance. Finally, I set the stage for the current project by describing trans voice training: the current clinical practices and objectives, as well as the resources and technologies that currently exist.

\subsection{Design Methods and Their Discontents}

PD's democratic origins in the labor movement have been well documented $[8,47]$. The methodology ideally seeks to characterize participants' existing practices, understand their needs and values, and translate that information into concrete recommendations. The tools of the trade here are typically qualitative, in-person, and limited in duration: in-depth interviews, focus groups, and surveys feed data into task analyses, personas, and design goals. These techniques ostensibly inject human needs and values into how technologies are produced.

However, recent work has shown how PD methods are part and parcel of exploitative systems, specifically in the context of HCI researchers working with underserved populations. Harrington et al. [40] examine issues of access, equity, and harm in the context of the "design workshop," a traditional PD method, in a predominantly Black neighborhood in the Midwestern United States. The design workshop, they describe, is a "spatially situated and temporally bounded coming together of participant groups and researchers to envision new design futures." Grounded in a community-based collaborative design methodology, they aimed to explore understandings of health and potential tools for health maintenance [39], and reflect on their process [40]. Ultimately, the workshops were fraught by legacies of institutional racism that could not be separated from the researchers' current work. One of their participants said, 'You know I'm old enough to remember when Northwestern would use us for studies but we couldn't get medical help. I remember. And this was after Tuskegee, knowing what they did. So, I have right to have my doubts." The researchers conclude by arguing for more equitable forms of PD through greater accountability, reflexivity, and acknowledgment of community history.

Rosner [62] describes the limitations of traditional PD by reflecting on how she designed a technology for knitters. She discusses how she began her research training with the "IDEO model" - empathize, define, ideate, prototype, test - and how she internalized those ideologies. Rosner argues that this creates and sustains a distance between designers and the people being designed for. In other words, participants tend to be briefly part of the design process, during which time their experiences and understandings of the world are extracted from them, and after which researchers apply that knowledge to further their own goals and agendas. The products generated from this process are prone to failure, and may not be needed to begin with [12, 40, 67]. Bennett [14] shows how the cornerstone of the IDEO model, empathize, denies the agency of disabled people. As a result, empathy exercises "may appropriate disabled people's techniques and experiences while 
rendering them non-designers through their disappearance. When designing for disabled users, such empathy work configures designers as different and isolated from the empathized." A distance between elite "innovators" and "others" assumes that a technological solution is needed [9], that these are two mutually exclusive groups of people, and that it can only be successfully built by the former [42].

Notions of agency and self-determination feature prominently in all of these accounts. Rather than being an abberation or accident, systematic denials of agency and power are part of HCI's founding ideology, and its ties to global capitalism and colonial histories [42]. However, scholars have begun to articulate alternative visions for design and evaluation. Asad elaborated "prefigurative design" as an explicitly political framework for "negotiating more just collaborations between researchers and community collaborators" [6]. By forging relationships focused on harm, healing, and transformation, Asad calls on researchers to "[co-create] counter-structures" that replace oppressive institutions. Harrington argues for a "reconstruction" in which HCI researchers address the "methodological challenges" to traditional PD arising from structural injustices [40]; similarly, Keyes et al. urges a "reorientation" of HCI towards creating "systems and spaces that exemplify the world we wish to see" [46].

Taken together, these provocations show a collective desire for a radical transformation in HCI. I argue that more methodological work is needed to accomplish this, work which must reckon with the material conditions that structure who precisely has the power to make decisions about the design and implementation of technologies. My goal is to provide just such an intervention, specifically within the context of building technology with and for trans people online. The following sections will describe this context in detail.

\subsection{Trans People and Technology Design}

A growing body of work in $\mathrm{HCI}$ centers on how trans people navigate, and are impacted by, existing technologies. For example, Haimson et al. [34] investigated how trans people navigate identity disclosures and gender transitions on social media. The authors show how trans users assert and navigate multiple identities across different platforms, and struggle to find in-group connections while simultaneously avoiding transphobic ${ }^{1}$ harassment. Prior research also implicates the social, cultural, and political phenomena that structure trans peoples' relationships with technology. Keyes [45] shows how automatic gender recognition (AGR) algorithms model gender as strictly binary (thus erasing non-binary people), and how these technologies are tied to gendered surveillance and police violence. Via qualitative analysis of interviews with trans people, Scheuerman et al. [63] describes six overlapping forms of harm: insider (within-group) versus outsider (out-group) harm, individual versus collective harm, and targeted versus incidental harm. The authors conclude that designers should "examine the very structures of their technological creations" and "access the rich history about intersectional marginalization... so they can understand how to sensitively fold them into new policies, practices, and software encodings." This valuable work disrupts reductive understandings of technologically-mediated transphobic violence at both the interpersonal and societal levels.

It is clear that no technological solution can address the multiple intersecting systems of harm described above. However, prior work has attempted to create targeted interventions for specific issues that transgender people face. In addition to this academic work, hundreds of commerciallyand freely-available apps have been created for trans users (a review of these is outside the scope of this paper; see [59]). I focus here on apps designed to support trans peoples' health and safety.

$\overline{{ }^{1} \text { Anti-transgender }}$ 
Beirl [11] describes the development of a bathroom safety and support app called GotYourBack, which provides a list of bathrooms near the user's current location, and uses crowdsourcing to provide a rating on how safe people felt in them. Designers first conducted semi-structured interviews with trans people, constructed an affinity diagram, and performed a task analysis. They then sketched prototypes and created a survey to elicit feedback on them, which was posted on "specialist forums"; the survey was also "shown" to trans people who had participated in their research. Their final design was reached via paper prototype evaluations (it is unclear whether trans people were included in this stage). Starks [66] conducted nine interviews with trans women and non-binary people of color, which informed the design of the safety app U-Signal. Participants shared that they wanted "no involvement" with the police due to misgendering and harassment; to address this, U-Signal allows users to share their device's location with their personal emergency contacts. The authors' describe their design process as a "human-centered approach," and involved semi-structured interviews. The initial interviews were with "one expert and one non-expert, which informed the initial design." Additional interviews were then carried out to understand participants' experiences with anti-trans violence, and obtain feedback on their prototype.

Outside of HCI, in the field of medical informatics, LeGrand et al. [49] describes Epic Allies, an mobile game designed for cisgender men and transgender women to support adherence to anti-retroviral therapy medications for HIV. In the game, users play as superheroes who are fighting monsters in the fictional city of "Medopolis." Periodic medication reminders and HIV-related information appear within the app; users can also track their dosages over time. Epic Allies was designed using the "Information, Motivation, and Behavioral Skills" model of behavior change; under this model, each app feature is geared towards one of those three pillars. The development team consisted of health researchers and an external "technology team" of consultants and programmers "with expertise in app design." The team created an initial concept on its own, and then conducted three focus groups of 20 Black men who have sex with men - the "target population" - to assess "adherence information, motivation, and behavioral skills needs," and obtain feedback and iterate on their prototype. An independent group of 7 men were recruited for task-based usability testing, to assess "participants' ability to successfully navigate the app, comprehend the educational content, and determine if they found the app to be engaging and relevant." A successful evaluation, the authors write, would support "the planned commercialization of Epic Allies" and "justify the costs invested." ${ }^{2}$

All of the above design processes prominently feature experts (whose expertise is rarely described and never questioned). Trans people serve the primary role of educating designers on their experiences, such that the latter may understand, empathize with, and design for the former. My intention here is not to disparage these research contributions; in fact, the first author's own work has been structured by similar ideas [3]. My goal is rather to emphasize the epistemological distancing between researcher and subject, which Bennett describes as "knowing the Other" [14]. In Epic Allies, this distancing is pronounced to the level of complete erasure: not only were trans women (and cisgender men) not consulted in the initial creation of the prototype, but the app was designed using focus groups, feedback, and usability evaluations with cisgender men only [49]. Although trans women were not mentioned in the design study, future stages of the project will recruit both cis men and trans women who have sex with men in a randomized control trial to determine the app's effectiveness [50]. The authors combine trans women and cis men together as part of the same target population, conflating them based on the gender of their sexual parters

\footnotetext{
${ }^{2}$ This mention of commercialization and economic justification appears out of place in an academic paper, and recalls Irani's argument that so-called "human-centered" projects serve "not as an orientation toward compassionate solidarity but rather as the mining of intimacies for projects of value creation."
} 
despite that they have unique needs and face different types of social oppression (which is a noted issue in HIV prevention/intervention research; see [60]). The authors do not indicate whether the design will be modified as a result of the inclusion of trans women, and only state that the app was developed "with input from the target population." These studies exemplify a trend in HCI and medical informatics research, wherein users are consulted simply as a means to a technological end, rather than as partners and co-creators. As Irani writes, designers often "invited users to exercise influence, but they rarely shared design control with users" ([42], emphasis in original) This paper asks: what possibilities are opened when trans people design for ourselves? What are the consequences when we are left out of the loop? What challenges might we encounter?

\subsection{Gender Presentation and Expression}

This section will briefly describe the context for the current project: the purpose and practices of trans voice training, as well as gender presentation and expression more broadly.

3.3.1 Trans voice training. A 2015 survey indicated that 62 percent of transgender women in the United States have either had voice training or currently want to have it [44]. However, that survey and other studies $[25,31]$ also indicated that trans people are impacted by high rates of societal discrimination, targeted assault and violence, homelessness, harassment from medical providers, denials of insurance coverage, and health disparities related to access to care. For trans people, voice can be closely tied to survival in terms of both mental and physical health. Trans people may find themselves held to a standard of what constitutes an appropriately gendered voice and be judged and addressed incorrectly, or potentially harassed and assaulted. Recent studies indicate that trans individuals are more likely to be victims of targeted assault and violence than the general population [44], and this fact can influence the way trans people perceive their own voice and their decisions to train it [3].

Dacakis [20] notes that the perspective and individual needs of the client must be included when evaluating outcomes. This focus relates to prior work examining constructions of identity in health and illness. Notions of identity are essential to trans individuals' health; voice can play an important role in both identity and health $[21,38]$. Despite this, it is important to recognize that voice is just one element in how an individual presents themselves. Furthermore, voice alone cannot predict how a trans person experiences the world, whether they are recognized as they desire to be, or their health outcomes more broadly (it may not be a factor at all for some individuals).

3.3.2 Queer and trans theory. The clinical literature on trans voice training paints a one-dimensional picture of voice training that flattens out the personal, social, and political complications of gender expression. Such studies often limit their inquiry to the effectiveness of voice training for "transsexuals" [17, 20, 22, 38, 72]. This language emphasizes a medical definition of transness [70]; in other words, the studies' recruitment pools appear limited to trans people who have undergone medical gender transition (hormone therapies and/or surgeries). Not only is it unclear whether and how to apply the results of the existing speech therapy literature to gender non-conforming and non-binary people, this also shows how the production of academic knowledge about trans people is structured by normative understandings of gender transition and expression.

Trans scholars have described how people must conform to particular social norms in order to "successfully" present as their desired gender [10, 64, 69]. For example, a trans woman might present in a more stereotypically feminine way than she otherwise would to secure access to gender-confirming medical services [64] or to avoid attention from law enforcement [3]. But what - and who - is lost through these normalizing forces, and who benefits from them? Everyone - not just trans people - are subject to regulatory gender norms, which are predicated on "the perceived 
deception underlying transgressive gender presentations" [10]. Casting gender non-conformity as deceptive has specific, sometimes lethal consequences for trans people [15, 71].

In his thorough analysis of vocal gender performance, Pennington writes that trans people are used to represent "the performed nature or constructedness of gender, which only serves to delegitimize their gendered identities while simultaneously rendering invisible and naturalizing the gender construction of cisgender people" [58]. Pennington reverses this harmful dynamic by focusing on the vocal (gender) performances of cis people, using transgender "passing guides" as an analytical tool. As Pennington writes, trans passing guides are often created by trans people to make "unconscious habits conscious again, so that a new set of gender vocalisms can be learned and internalized" [58]. The existence of these community-made guides can be seen as tools of assimilation, affirmation, and/or survival; trans people vary widely in whether, how, and when they adopt gendered norms of speech in their everyday gender expression [3]. As Haimson [34] describes through the concept of liminality, trans people in transition "exist multiply," holding "multiple, fragmented, and complex" identities both online and off. Haimson urges technology designers to account for this complexity in their work [36]. When it comes to voice training technology, however, the current options largely reduce vocal gender transition to an uncritical reproduction of gendered stereotypes $[4,69]$.

3.3.3 Existing voice training technologies. Unlike passing guides, trans voice training apps are often for-profit and developed by clinical speech therapists who are cisgender, white European/American women [51, 52]. As shown in prior work, these apps often perpetuate normative, racialized, and classed gender categories [4]. EVA [51], for example, features a stereotypical pink/blue color scheme (depending on whether one downloads the version for trans women or trans men, respectively), and uses photographs of apparently white, affluent, gender-conforming people as background images. Functionally, the software assumes a singular and unchanging transition goal: a "female" or "male" voice; user customization is non-existent. The speech therapist's personal brand also strongly influences their app's design and functionality, and thus the user experience. In the app Christella VoiceUp, the app encourages users to "find out how [therapist] Christella [Antoni] can help you" using the "Antoni M2F Voice Change Model" [52]. The identity and qualifications of the speech therapist are highlighted through images, text, and video lessons (available through in-app purchases). One app features an "About" page with her photo and qualifications [51]. As the deliverer of information, the speech therapist positions herself as an ambassador to the user's gender enlightenment.

These clinician-driven apps differ significantly from other offerings. Voice Pitch Analyzer [61], a popular free and open source voice training app for Android smartphones, allows users to read a passage of text and see their pitch in comparison to normative gendered ranges. Although the for-profit apps described above also provide this feature, Voice Pitch Analyzer is free (of charge, and of gendered color schemes). It does not assume that the user has a particular gendered goal, nor can the user specify one. Additionally, trans people interested in training their voices use a number of no-frills apps that are not specifically designed for trans people, but that simply provide a visualization of the pitch or resonance patterns of the user's voice.

As I have shown in prior work, trans participants described how their willingness to use a system hinged on whether or not it interfered with their ability to make their own decisions about their lives [3]. Normative gender roles and transition narratives influenced how participants thought about their own transition, and participants talked about how they are often marginalized in - and distrustful of - social and medical institutions. Potential designs aiming to support trans people must understand the systematic denials of agency that result from living in a transphobic society, thereby facilitating the creation of artifacts that respect these concerns. 


\section{PROJECT SPECTRA}

With the above context established, this section will describe Project Spectra, a collective of open source developers working to build trans voice training software since July 2018 . We begin by describing the platform and practices under which we worked, what we created (along with why and how), and reflect on the process through the lens of community-based collaborative design We end by discussing what our work means in the context of the sociopolitical systems described above. A full elaboration of Project Spectra's functions and features is beyond the scope of this paper (for the source code and app, please see [2,68]). We will touch on some of the major design elements here only insofar as they shed light on the design process.

\subsection{Autoethnographic Data Collection}

This paper draws from my design memos to understand and communicate how Project Spectra works, and my role within it. Autoethnography has been previously used to reflect and report on research processes, to push towards "a more just experience" for research collaborators [7]. With this intention, this paper uses an autoethnographic approach, which ultimately seeks to "describe and systematically analyze personal experience in order to understand cultural experience," and which "acknowledges and accommodates subjectivity, emotionality, and the researcher's influence on research" [27]. Contrasted with ethnography writ large, Ellis writes, autoethnography does not involve a researcher observing and documenting an "outsider" culture, but rather writing about experiences "that stem from, or are made possible by, being part of a culture and/or by possessing a particular cultural identity."

While I worked with Project Spectra, I created 28 "design memos," which were written and stored in Google Drive and accessible to all Project Spectra members. Early on, entries were recorded within this document on a weekly basis. Beginning in January 2019, memos were recorded on an ad-hoc basis (sometimes multiple memos per week, as needed). The content of the memos cover all aspects of the design process: technical/code issues, meeting notes, critical decision points, questions and discussions related to voice training pedagogy and practice, speech processing algorithms, and interface design/sketches. I also used the document as a space for theoretical reflection on our design process, its successes, and its challenges. I consider these design memos as autoethnographic field notes, which document events as I experienced them, and as "in-process memos," which "[allow] the fieldworker to develop analytic leads and insights" [28].

I use these memos in an effort to provide an honest and respectful description of our work as I experienced it, as well as to inform researchers and practitioners interested in designing transcompetent software, trans voice training software, or online community-based collaborative design more broadly.

\subsection{Membership}

Invitations to the Project Spectra team were extended when any person expressed interest in joining; no technical or other experience was required. Potential members were contacted by current members through social media networks, primarily Twitter and Discord ${ }^{3}$. Discord is a free, proprietary chat client primarily geared toward gamers. Users can create "servers" containing any number of chat channels, where members of the server can communicate via text, audio, or video Servers operate independently, but Discord users can be a member of multiple servers at once.

After initial contact, I typically met with the person (via phone, video chat, or text) and discussed their backgrounds and goals, interest in the project and its current status, and ultimately asking

$\overline{{ }^{3} \text { https://discordapp.com }}$ 
whether they would like to be involved and in what capacity. Either during or prior to this conversation, I invited them to look over some of our documentation, specifically our Principles and Goals (see below). I also explained the following:

- No amount of participation is required or expected by virtue of their joining the team.

- I am a graduate student who is doing this work as a component of my thesis project.

- Our primary goal is to create this voice training app, but we also strive to create an environment of mutual learning and support.

Project Spectra members brought our own expertise and perspective to bear on the project in the time and capacity we had available. As such, some members focused on contributing design mockups, speech analysis algorithms, front end development, big-picture feedback, or some combination of these. We also asked several people to provide feedback and guidance to the project either in one-off or recurring timepoints, but they did not participate in regular communications. These included speech therapists, HCI researchers, and trans people interested in voice training.

Although this project was approved by Northeastern University's Institutional Review Board, written informed consent was not required to be a part of the Discord channel and to participate in group activities, and was not obtained. As a result, this paper does not reproduce quotations from members. Information about group members, demographic or otherwise, was not formally collected. Members also did not need to share their names or other aspects of their identity - though they sometimes did - to participate in the group. Members did not receive any compensation for their participation in group activities. All communications with group members and on our public channels were in the English language.

In March 2020, current Project Spectra members were informally polled and asked to share details about their age, gender, racial identity, and extent of their technical skills. Nine members of the group responded to the poll. The mean age of respondents was 29 (SD $=6.91$, range $=$ 21-40), participants' gender identities included transfeminine, non-binary, and gender questioning. Respondents identified themselves as white or Caucasian (3), Asian (2), mixed/multiracial (3), and East Asian (1). In terms of economic class, responses included upper middle class, to middle class, and working class. Finally, respondents were asked to rate the extent of their technical skills from 1 (no coding experience) to 5 (professional/expert programmer). The mean score was $3.8(\mathrm{SD}=1.2)$.

\subsection{Collaboration Tools}

We used a combination of freely available tools to collaborate online. Because there was no coherent set of tools or platforms determined at the outset, we explored potential options together, sometimes discarding them completely if they stopped being useful or suitable to our needs. Project Spectra's main workplace is Discord, which was chosen because two large ( $>1000$ members) trans voice training communities already existed there. Our server contained general channels such as "\#workspace," "\#design," and "\#engineering," and also separate channels for meetings, such as "\#2019-04-26-general." Members also communicated outside of servers through direct messages. Between July 2018 and November 2019, we convened six general meetings, two code review meetings, and two design meetings. We also met during loosely scheduled "coworking hours" in which members could drop-in and drop-out as they liked.

In addition to communicating asynchronously on Discord, we used Google Drive to share documents, sketches, mockups, notes, contacts, and other resources. We also used Google Forms to create and share user testing surveys. For code collaboration and version control, we created a Github organization ${ }^{4}$, which houses public code repositories. Through Github, we also logged

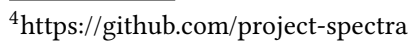


issues and bugs and assigned tasks. All team members had - and continue to have - full ownership access to the Github organization.

We started by using Sketchboard ${ }^{5}$ for digitally sketching potential screens and speech data visualizations, and for creating flowcharts for how those screens would connect to each other. We abandoned Sketchboard and later switched to Whimsical ${ }^{6}$ for flowcharting, because we found the automatic layout generation to be cleaner and less cluttered (see Figure 1). We also used the freely-available tool Adobe $\mathrm{XD}^{7}$ for wireframes and final digital mockups because one of our members was already familiar with it. Our mobile app was implemented in NativeScript-Vue ${ }^{8}$, a framework for cross-platform development based on JavaScript; see Figure 2 for a comparison.

Because Project Spectra members are located all over the world, some established participatory design activities - such as in-person design workshops and focus groups - were not feasible. The following sections use the first author's design memo documents (in which I recorded major decision points) to illustrate our design process.

\subsection{Principles and Goals}

Project Spectra crafted a "Principles and Goals" document in August 2018. This document was then presented to all new members when they joined the group 9 . Its full text is below:

**This is a living document. As our members grow, this document should too. Feel free to suggest additions or edits!**

We are a group of independent developers and designers seeking to build free \& opensource software for voice training. While we believe that anyone, regardless of identity, should be able to use and benefit from our work, we are specifically interested in supporting the self-determination of transgender \& gender non-conforming people. As individuals who have pursued voice training ourselves, we want to create software that we would want to use. However, we also recognize the limitations of our own perspective and background. Therefore, we intend for our software to:

- Respond to the concerns, needs, and lived experiences of other trans people.

- Put users in control of what aspects of the voice are trained, and to what end goal.

- Incorporate and take advantage of current research and clinical practices, as well as the expertise within our community.

- Not marginalize users, or further harmful notions or expectations, with regards to gender, race, sexuality, nationality, ethnicity, and ability.

- Be accessible to as many individuals as possible.

- Be freely available and open source, in perpetuity.

We intend for ourselves to:

- Foster meaningful community participation, both digitally and in-person, around the design and development of the software.

- Co-create a safer space, in which all contributors to our projects are treated with dignity and respect.

\subsection{Programming Framework}

Early in the design process, our team considered whether to begin our development by splitting off of (or "forking") an existing open source voice training app called Voice Pitch Analyzer [61], or

\footnotetext{
${ }^{5} \mathrm{https} / / /$ sketchboard.me

${ }^{6}$ https://whimsical.co/

${ }^{7}$ https://www.adobe.com/products/xd.html

${ }^{8}$ https://nativescript-vue.org

${ }^{9}$ We also later adopted a version of the Contributor Covenant [26], a more formal code of conduct used in FOSS teams
} 


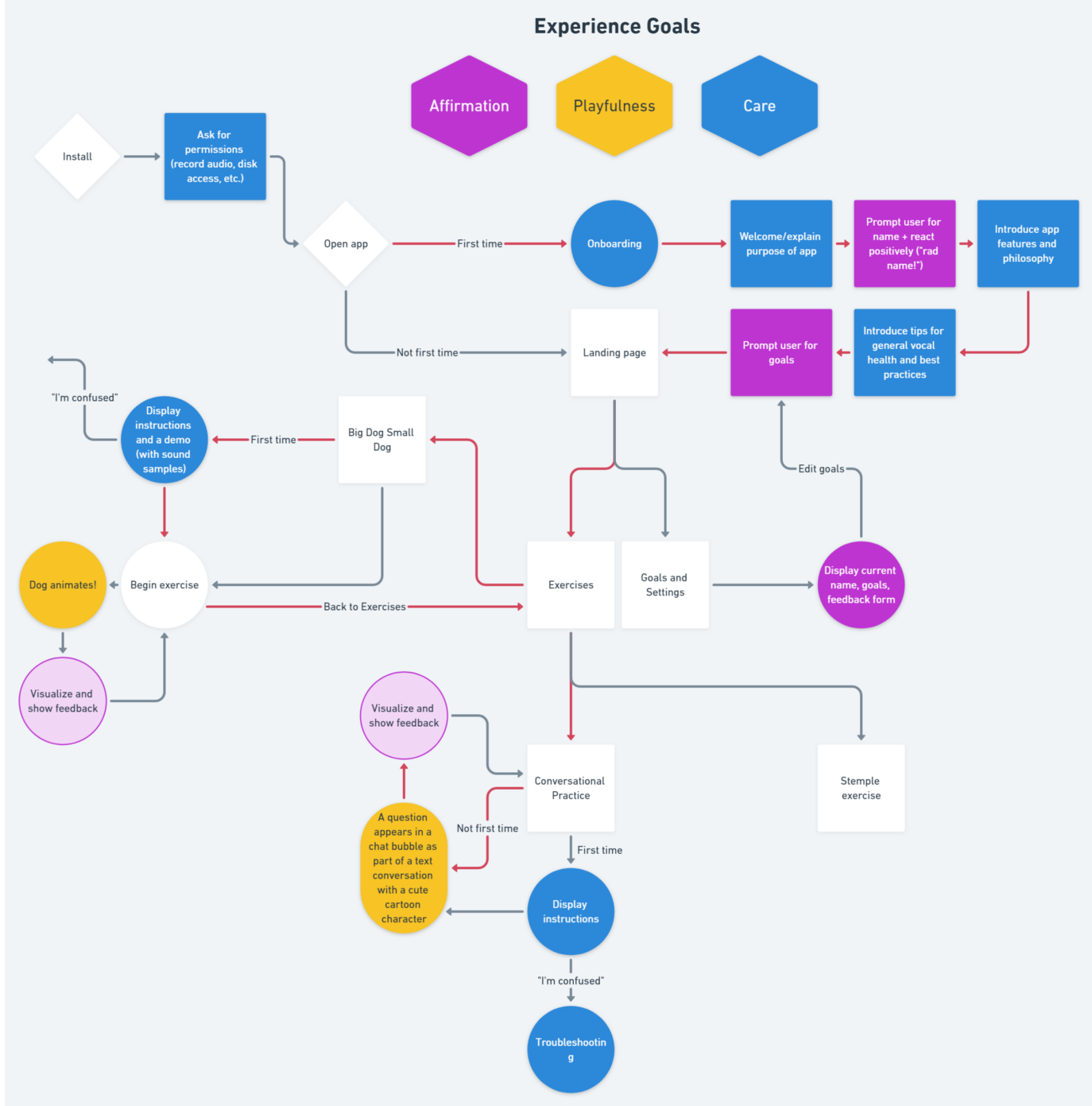

Fig. 1. App flowchart created using whimsical, May 2019. Each app screen or interaction is color-coded with respect to our Experience Goals (see section 4.6). In addition, we use red arrows to highlight the "happy path" that users would ideally follow upon opening the app for the first time.

to start from scratch. In Design Memo 3, dated the week of August 3-10, 2018, I summarized our discussion in the form of pros and cons for both options (see Table 1).

Critical to our decision to start from scratch were that "more people will have access." VPA, the existing open source voice app, while well-designed and useful, is only available on Android. This decision was difficult, though, because VPA has a "massive" current user base who we could access for testing out new features incrementally. Also, the existing app is first and foremost a pitch detector; adding or elevating non-pitch related features could "disrupt" those users who do find those features essential. The existing open-source codebase was not just Android-only, but Android-specific - i.e., very little (if any) of it was platform-agnostic. Choosing VPA meant being 

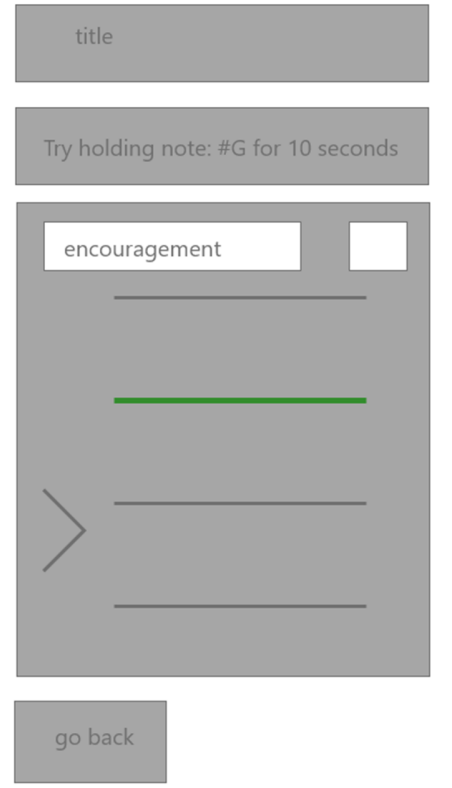

Fig. 2. Comparison of one app screen through development stages: wireframe (left), mockup (middle), and implementation (right).

stuck with Android for the forseeable future, and that major refactoring and scratch work would have to be done to port to other platforms.

This early decision solidified Project Spectra's prioritization of access, as described in our Principles and Goals document (see section 4.4). Ultimately, working off an existing app would not only have constrained current VPA users' autonomy - as they may have to deal with unwanted changes to an app they were familiar with - but also constrain us as developers. We may have found ourselves limited by the voice training approach of the original app, and tasked with "fitting our ideas into" a design space where our ideas were incompatible. Specifically, we needed to contend with the fact that "we don't think pitch is that important," an idea backed up by the clinical literature [17]. In this way, our team resolved to carve out a new space for an app that would offer something that the currently available options did not. As a result, we chose to use NativeScript-Vue, a freely available framework that compiles to both Android and iOS.

\subsection{Experience Goals}

In January 2019, one of our group members decided to lead a discussion on Discord to formalize the project's "Experience Goals," to serve as guideposts throughout our design process. In so doing, we formalized our values in a manner similar to our Principles and Goals document, but more focused on the design artifact, and the experience we wanted to evoke and support. From our conversation, we distilled three goals, which were presented and finalized at our general meeting on 15 February 2019:

- Affirmation. Users must feel supported and represented, and feel that their choices and identities are respected and validated by the app. 
Table 1. Summary of our group discussion about the pros and cons of starting our own app from scratch.

\begin{tabular}{|c|c|c|c|}
\hline \multicolumn{2}{|l|}{ Start from scratch } & \multicolumn{2}{|l|}{ Fork VPA } \\
\hline PROS & CONS & PROS & CONS \\
\hline $\begin{array}{l}\text {-As a result of the } \\
\text { above, more people } \\
\text { will have access to } \\
\text { the app in the long- } \\
\text { term }\end{array}$ & $\begin{array}{l}\text {-Frameworks are } \\
\text { potentially unstable } \\
\text { (Flutter, for exam- } \\
\text { ple, is still in early } \\
\text { development) } \\
\text {-Coding may be } \\
\text { slower and more } \\
\text { difficult since some } \\
\text { members of the } \\
\text { team may have to } \\
\text { learn a new lan- } \\
\text { guage/environment }\end{array}$ & $\begin{array}{l}\text {-And also greater ac- } \\
\text { cess to new users in } \\
\text { the short-term }\end{array}$ & $\begin{array}{l}\text {-Since our planned } \\
\text { changes are fairly } \\
\text { radical, they may } \\
\text { confuse/disrupt } \\
\text { users (but this could } \\
\text { be solved w/ an } \\
\text { opt-in beta test?) } \\
\text {-Only Android users } \\
\text { will have access to } \\
\text { our app }\end{array}$ \\
\hline $\begin{array}{l}\text {-Don't have to deal } \\
\text { with fitting our ideas } \\
\text { into an existing app } \\
\text { design/voice training } \\
\text { approach }\end{array}$ & $\begin{array}{l}\text {-Q: Will we be able } \\
\text { to do all the calcula- } \\
\text { tions we need to do in } \\
\text { whatever new frame- } \\
\text { work we choose? }\end{array}$ & $\begin{array}{l}\text {-Have access to [VPA } \\
\text { developers] as a sup- } \\
\text { port }\end{array}$ & $\begin{array}{l}\text {-Given that we don't } \\
\text { think pitch is that } \\
\text { important, it may } \\
\text { make absolutely } \\
\text { no sense to retain } \\
\text { current VPA features; } \\
\text { which would disrupt } \\
\text { users who do like } \\
\text { those functions if } \\
\text { our changes do get } \\
\text { pulled in }\end{array}$ \\
\hline
\end{tabular}

- Playfulness. The app should be welcoming and encouraging to users without seeming condescending or childish.

- Care. Users should never feel strained, overworked, or overwhelmed. The app should project a sense of clarity, restfulness, and trust.

Finally, we concluded that:

"Design should focus on simple workflows with clear objectives and interpreted feedback. User experience should be as customizable as possible, with the user in control of how the app interacts with them and refers to them, and this should be alterable at any time. The ultimate experience goal of this software should be a 'warm fuzzies' moment!" 

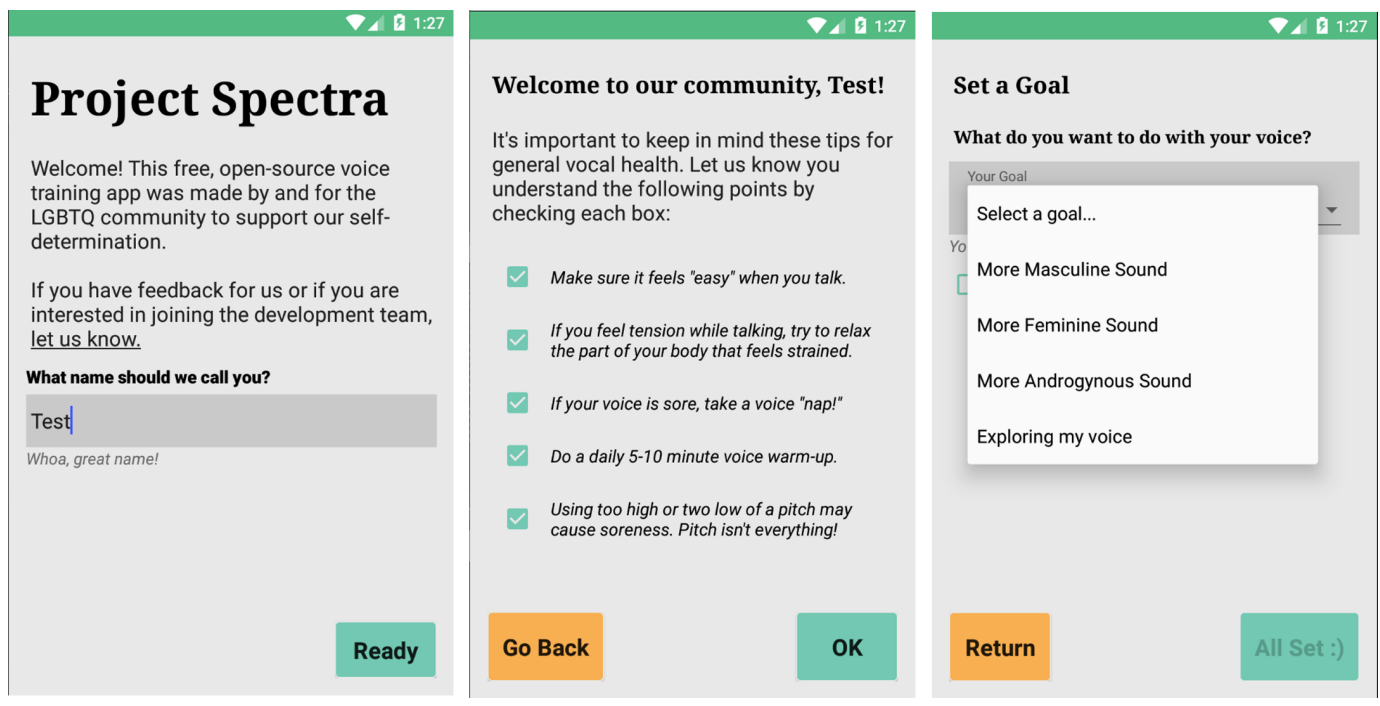

Fig. 3. Onboarding screens for the Project Spectra app, which show how our Experience Goals of "Affirmation" and "Care" were implemented. Users can choose and change their name and goals (left and right) at any point while using the app, and the app emphasizes vocal health and wellbeing over achieving a predetermined gendered voice goal.

The "Affirmation" goal describes the agency we wanted users to have, which counteracts the social forces against trans people (see section 3.3). As shown in Figure 3, our app prompts users for their names and voice goals when they first open our app. Rejecting normative ideas and narratives about linear gender transitions, we also provide easily accessible options for users to change their names and goals at any time. On the goal selection screen, users choose whether or not to compare their progress against a specific pitch goal, which is selectable in lieu of, or in addition to, masculine/feminine/androgynous options.

Our Experience Goals recognized the often fraught relationship trans people have to voice training: that it can be extremely stressful, time-consuming, confusing, and overwhelming. With the "Playfulness" goal, we described our aspiration for the app to counteract this problem by being "welcoming and encouraging" but not "condescending or childish." To fulfill this goal, we considered designing a cartoon character or mascot to make the app more fun, and periodically presenting supportive messages to users. Months later, these ideas would make their way into our final prototype.

In February 2019's general meeting (and during the preceding weeks), we also concluded that the app needed more voice exercises and an increased emphasis on vocal health. Voice strengthening exercises (also known as Vocal Function Exercises, or VFEs) can benefit users regardless of their specific goals ([29]). The exercises encourage people to use a well-supported voice that is less prone to strain. Our conversations with speech therapists confirmed that vocal health should be kept "front and center," and as a group we considered ways to encourage users to strengthen their vocal cords and take breaks. As a result, we reflected the change in our flowchart (see Figure 1), which color codes elements of the design by our Experience Goals. This translated directly to their incorporation in our digital mockups, and finally in our prototype design. This decision directly corresponded to the "Care" goal, because it would work against users feeling strained and overwhelmed. 
The collective articulation of our goals strongly influenced the design process. Not only were these goals community-sourced, in that they came from queer and trans people directly, they were also community-implemented.

\subsection{User Testing}

Our Principles and Goals document stated that "we want to create software that we would want to use. However, we also recognize the limitations of our own perspective and background." To help us make design decisions, we repeatedly reached out to external groups and individuals whose perspectives we valued. This included trans people who are interested in voice training, speech therapists, and the individuals who responded to our user testing surveys. I also consulted members of my dissertation committee, and presented their thoughts to the group as suggestions rather than directives.

The Project Spectra team created and released two user testing surveys: the first covered the onboarding flow $(n=20)$, the second covered voice training exercises $(n=9)$. The surveys were sent to two Discord channels populated by trans people interested in voice training $(\mathrm{n}=$ approximately 1500 and 1000), two Slack channels of HCI research groups at Northeastern University ( $\mathrm{n}=20$ and 31), and several trans technology researchers who were interested in the project. As I wrote in Design Memo 16 (dated 25 April 2019), we intended to get a "mixture of responses" from "potential users and from design/technology researchers." An internal survey $(n=5)$ was also released to Project Spectra group members regarding the design of the app's cartoon character mascot.

During design meetings in early April 2019, we finalized the introductory text of our first user test:

Project Spectra is a collective aiming to create free, useful, and respectful voice training software for transgender and gender non-conforming people. Our work is guided by our own experiences, but we seek input from others to make the best app we possibly can. Your participation in this test will help us do that. Thank you very much for your time and attention :)

As a tester, you will take the role of someone who has just installed the "Project Spectra" app. You are interested in training your voice, but you do not know much about it.

The test should take less than 15 minutes.

Things to know about being a tester:

(1) There are no right or wrong answers to the test - we are testing the software, not you

(2) Please share all your thoughts (your "inner monologue"), whether they are positive or negative

(3) You will not hurt our feelings by giving your honest opinion on our work

Thank you again!

Project Spectra Team

User tests contained a series of mockup screens; each screen was presented one at a time alongside a series of questions, such as "What do you think you need to do here to move forward in the application?" or "What can you tell about your goal from this screen?" All questions solicited open-ended text responses. In our general meeting on 27 April 2019, we summarized and discussed preliminary results from our first tester. In the notes, I wrote that:

"the UI patterns we employ such as entry boxes, drop downs, tip acknowledgement and progress indication are very clear and immediate. also on the pros side, most respondents felt that the tips area in particular was very informative and delightful. I would say it's our biggest area of positive feedback...on the constructive criticism 
side, some users felt that the encouragement phrases we selected were a little patronizing. this is directly against our goal of being supportive to our users, so this is the biggest area in need of adjustment" (emphasis in original text)

As a result of the feedback our group received over the three surveys we sent out, we increased the saturation of the color scheme, clarified the text displayed to be "less jargony," and made the pitch goal visualization more intuitive (Design Memo 20, 31 July 2019).

\subsection{External Consulting}

In Design Memo 13 (dated 26 February 2019), I reproduced the notes from my meeting with a Boston-based speech therapist, whose opinion on how we should proceed diverged from our group's current trajectory. One component of voice modification for some trans people is larynx training, in which people alter the position of their larynx through repeated exercises. The trans voice communities online, particularly on Discord, commonly recommend the exercise "Big Dog Small Dog." In this short exercise, one slowly transitions from panting like a small dog (near the front of the mouth, the larynx should elevate) to a big dog (near the back of the mouth, the larynx should descend). Because the exercise is so popular in our group, I had already added it to the app; in Design Memo 5 (dated November 2018), I described the trans voice training community's general understanding that: "Raising [the] larynx leads to a brighter sound, reducing space in the vocal tract. Conversely, lowering the larynx leads to a darker sound. Testosterone has an effect on the vocal tract which lowers the larynx - for voice training in trans women, the intention is to correct for this." However, the speech therapist I spoke to believed that the larynx should never be held in one position. In my notes, I summarized the speech therapist's opinion as the following:

"The larynx should not be held in a certain position - this requires extra muscle use and could lead to strain. Instead, the larynx should rise and fall naturally. However, [the speech therapist] doesn't insist that we drop the exercise from our plan. She says there's still a lot of unknowns in this field and that her views are open to change. She has seen people for whom larynx manipulation works, but she has also seen it hurt people."

This exchange sparked a discussion on our Discord channel about the differences in voice training practices between clinicians and trans people. Trans people who have speech therapists do not always agree with them [3]. As our collaborator admitted, how trans people ought to train their voices is not an exact science; as section 3.3 describes, it is also fraught with gendered norms and expectations. The clinical field is relatively young (the most comprehensive text for speech therapists working with trans people was first published in 2006, with subsequent editions released in 2012 and 2018 [1]). Our group had to contend with this social context, and ultimately decided that we wanted to privilege the lived experiences, practices, and knowledge of trans people, rather than institutional knowledge about us.

Prior research has highlighted trans peoples' distrust of research and medical institutions - and with good reason [30] - and are actively forming their own knowledge bases drawing from our own experiences. This does not mean that the medical standpoint is always wrong (or that trans people are always right). Medical and health apps often position themselves as authoritative sources, which obscures the contested and socially situated aspects of the information being presented. I named this issue in Design Memo 13, and asked: "Is there a way to work against this in the design of the app? A way of naming this issue so that we are fully transparent to our users?" This question, like many others that came up during our process, was not addressed in our final (albeit functional) prototype. 


\subsection{Challenges Relating to the Group's Direction}

Stress related to my graduate school timeline led to adverse consequences within the group. Late in the design process, I needed a working prototype of our app so that I could move forward with my dissertation work, as per the requirements of my program. In Design Memo 18 (dated 18 June 2019), I wrote: "I had a meeting with my dissertation committee today. They're saying that in order for me to graduate on-time, I need to have a usable prototype done by Thanksgiving (to leave adequate time for pilot testing and debut-ing)." I then end by saying, ominously, "That's about 5 months from now."

That fall, the group experienced an influx of new members through snowballing; new members invited their own connections, and the group's size increased from 10 to 20 members. One of them had an idea for a new app, separate from the one described in this paper, and began to recruit team members towards creating it. In Design Memo 25 (2 October 2019), I wrote:

"I recently experienced an interaction on the Discord that left me evaluating how I respond to people in the group. Do I feel threatened or defensive when members come forward with ideas that deprioritize work that I spearheaded (the current Spectra app)? I was eventually able to speak more reasonably about how much I valued that member's initiative.

Edit (10/8): Members also suggested that the short-term priority should be to help me finish my degree, and the long-term goal is to make something beneficial to the community. This affected me. I remember being up late and looking at my phone in the dark, because of the time zone differences, right as I was getting ready for bed. I ended up sitting up and typing responses on my phone in the Discord app, saying that making something beneficial to the community is why I am here too - not just to complete my degree. Here is what I said: 'As far as my degree goes, really don't worry about it! That's for me to deal with - I don't want my academic requirements getting in the way of what you all want to do...'

However, that doesn't change the fact that the community of Project Spectra was built specifically around the constraints of my degree."

An autoethnographic approach allows us to examine this situation as data, not just about me, but also about the organization. From this interaction, it is clear that: (1) at least one member felt that the group's priorities should shift; (2) I felt worried that this would prevent the group from reaching a suitable stopping point for the existing Spectra app (which would put my dissertation timeline in jeopardy); and (3) this worry caused me, initially, to react negatively to the group member's initiative, despite that I had dedicated substantial effort to getting group members to participate over the past year. As I reflect in the following sections, this dilemma stemmed from how academic design research is structured.

\section{DISCUSSION}

Our design process involved a combination of techniques from participatory design (PD) and free and open source software (FOSS). I argue that in such localized contexts (such as a group designing with and for itself), standardized and hierarchical models for design begin to lose meaning. As a collective, we moved toward a bottom-up process where collective decisions dictated what methods to use and when, how user research is conducted and interpreted, and what implementation decisions are made. That said, the work was anything but straightforward: there were no shortage of tensions, challenges, and power dynamics at play in the team, as reviewed below.

Throughout the design process, team members brought their unique experience and expertise and acted on it. Contributors freely made significant decisions about (and contributions to) our project 
without needing approval or permission from a hierarchical system. Our always-connectedness allowed spontaneous yet critical decisions to take place as needed in the moment, but differences in timezones meant that not everyone was available and online when spontaneous interactions were taking place. Open-endedness was therefore a drawback as well as a strength.

Taking our group as a whole, I want to ask whether our daily practices aligned with the vision and intention of a non-hierarchical, community-based design effort. In so doing, I answer Rosner's call to look at moments of breakdown and difficulty as an important source of data [62]. Taking a reflective stance, I inspect my own complex positionality and multiple roles as a trans person (and hence a target user), an organizer and member of Project Spectra, and an academic and HCI researcher.

\subsection{Relationships to Existing Groupwork Models}

In this section, I describe our groupwork practices in relation to previously described frameworks. Rather than name our process as yet another model, I argue that it is important to flexibly choose collaboration tools and methods, specific to the domain in question, that maximize contributors' agency and ownership over the project.

Free and open source software (FOSS) development cultures have been the subject of extensive research. Ducheneaut [23] conducted an ethnographic study of Python contributors through their listserv email communications. By following individual developers' trajectories, he found that "successful contribution to an OSS project is much less about technical expertise than about the construction of identities." Building an identity for oneself involved attaining status through material/technical contributions, as well as gaining the approval of a network of allies (particularly of "core members" or leaders of the project). Python contributors were thus socialized into complex norms of participation, both implicit and explicit. No "hand holding" or coaching was offered to newcomers; rising through the project's hierarchies required both technical skill and political acumen. Deucheneaut writes:

"Despite the rhetoric surrounding Open Source, which basically argues that 'anybody can contribute,' it seems instead that only those few participants who have managed to define and present themselves as 'software craftsmen' eventually reach the status of developer in a project.” (p. 362)

Deucheneaut's analysis tracks individual contributors - who are assumed to be men, judging by his exclusive use of masculine identifiers - as they compete for in-group status. Other research on FOSS development culture adds nuance to this narrative by describing the organic formation of subgroups [16] and tightly-coupled teams focusing on smaller tasks within a project [55]. The barriers to participating in open source teams are cultural as well as technical, and uniquely disadvantage women (see [54] for a comprehensive analysis). Wubishet [73] argues that "the very same possibilities of participation that FOSS culture and practice provide often impede the participation of lay users." Because technical proficiency is required to participate, FOSS systems are seldom designed with end users in mind; design requirements are assumed to be "generally understood," leading to a discounting of the experiences and needs of end-users, in turn leading to poor usability. The authors suggest that "technically capable and active core team members usually have more authority and decision-making rights" and that end-user critiques are not taken as seriously. As a result, FOSS software tends to be developed "by experts for experts." Wubishet and colleagues then suggest that participatory design (PD) can help FOSS projects overcome this problem, because PD focuses on "mutual learning" and "active cooperation" between developers and users. Because the key activities - workshops, brainstorming, and prototyping - all typically take 
place in-person, designers can immerse themselves in a setting and build substantive relationships with target users.

Although participants can directly influence the design process without needing any technical experience, the power dynamic between researcher and participant is left unaddressed; "experts" remain the primary decision-makers, just as they do in FOSS (see Section 3.1). Under both frameworks, developers have the last word in what ends up in their designs. Many decisions that go into development in practice (such as how to represent or calculate a variable in the back-end) can only be made by developers can make in the moment, and PD models obscure them, marking them not as important as the user experience. The commons based peer production (CBPP) model attempts to address these issues. It focuses on "autonomy, democracy, justice... a shift from consumers to users, doing more for and by themselves, and in a loose association with others." According to Benkler [13], such projects have the following major characteristics:

- Decentralization: authority to act does not rest with a central manager or organizer

- Social cues and motivations coordinate joint action, rather than corporate hierarchy/stakeholders

- The project must be modular

- The modules should be fine-grained

- There should be low-cost integration mechanisms

In Design Memo 8 (dated 25 January 2018), I began to reflect on how we work, and how that influences the artifact we are creating and our relationships to each other. I wrote:

"I believe that the CBPP model has direct relevance to Project Spectra, and can guide us in our development. I think our project is modular in that there are clear components: algorithms/speech analysis, back-end programming, UX/front-end design, voice exercises. It should be easy, for example, for a person with low technical experience to follow what's happening with the project and contribute their ideas. Traditional PD techniques can be applied during adoption and customization (evaluation phases), because a functional system is at hand for end-users to work around. I got an email from someone who really wanted to help out... I did a short interview with them to hear about their experiences, and offered to keep in touch about prototypes (they have an Android phone, so it should be easy to get that working). But what else could they help with? What are other 'integration mechanisms' that could allow this person to contribute ideas?"

Later, in a memo written on April 4, I attempted to more concretely address how our project worked in relation to PD, FOSS, and CBPP (see Table 2). I wrote, "What model do we most conform to in practice? Is this what we want? Although I would aspire to a CBPP model, I think that our project contains elements of all three models." Some of the qualities of the project described in Table 2 arose despite my intentions. For example, team members referred to me as the "project lead" even though I never claimed that title for myself. I inhabited that role based on my day-to-day actions: coordinating and facilitating meetings, introducing new team members to the project (I am their first point of contact), and keeping documentation in order. I am also a contributor to the code and the design, but those do not conform to a particular role within the project (we do not have assigned roles in our team). I did not directly assign tasks; rather, things got done based on the motivations, skillsets, and collective decision-making of team members. My role included scheduling and facilitating meetings, drafting agendas, creating paper and digital mockups, analyzing user survey results, and writing code. Beyond that, I internalized a sense of what people's capacities were, holding in my memory their general interest in the project and what they wanted to work on, trying to create opportunities and situations for people to get involved and feel included, while at the same time not being pushy. This demanded significant interpersonal skills and sensitivity; and I did 
Table 2. Comparing groupwork models in the context of Project Spectra. CBPP is commons-based peer production; PD is participatory design; FOSS is free \& open source software.

\begin{tabular}{|l|l|}
\hline Quality of Project Spectra & $\begin{array}{l}\text { Associated } \\
\text { work model }\end{array}$ \\
\hline $\begin{array}{l}\text { Modular components (algo- } \\
\text { rithms/speech analysis, back-end } \\
\text { programming, UX/front-end design, } \\
\text { voice exercises) }\end{array}$ & CBPP \\
\hline $\begin{array}{l}\text { Not profit-motivated; most decisions } \\
\text { made collectively }\end{array}$ & CBPP \\
\hline $\begin{array}{l}\text { Non-technical individuals consulted us- } \\
\text { ing interviews/user testing/other meth- } \\
\text { ods, some of which are face-to-face }\end{array}$ & PD \\
\hline $\begin{array}{l}\text { Organized/managed by an central indi- } \\
\text { vidual (me) }\end{array}$ & PD \\
\hline $\begin{array}{l}\text { Open-source; team members involved } \\
\text { in implementation; asynchronous }\end{array}$ & FOSS \\
\hline $\begin{array}{l}\text { Some modules require high levels of } \\
\text { technical skill and background knowl- } \\
\text { edge }\end{array}$ & FOSS \\
\hline
\end{tabular}

not always do this well. I felt a desire to act as a support for team members who were having a hard time (for example, if someone cannot make a meeting because they are going through something, I would reach out to ask how they were doing and if I could support them). Interpersonally, it was difficult to maintain the level of care that I wanted to exhibit, especially if I did not know the person very well. Though I believe we trusted each other, we never met in-person and we communicated primarily over text channels (with the occasional voice and video call).

Taking the above reflections together, it is clear that I was acting as a central organizer, despite my intentions not to. As the next section elaborates, this had several cascading effects on our design process.

\subsection{Examining A Moment of Breakdown}

Feminist epistemologies consider experience an important source of data; attending to "moments of breakdown" and difficulty can serve as a window into the design process [62]. By any measure, the experience I documented in Section 4.9 was a moment of breakdown. Rapidly coming up against a deadline, I felt pressure to meet the expectations that my dissertation committee and funding sources laid out for me. Texting late into the night on our Discord channel, I did not think about how to respond appropriately to this team member. I felt that I had failed because another member suggested that the group should help me finish my degree before making something beneficial to the community. I felt ashamed because my behavior was impeding the very principles to which I had aspired: to encourage open collaboration, to cede control and dissolve hierarchies It is interesting to consider how differently I might have reacted if this interaction had occurred during an in-person design session; I might have responded more thoughtfully, and I might have 
known them and their goals and interests better. On the other hand, it seems less likely that this interaction would have occurred at all: focus groups and other in-person design exercises rarely allow participants to act collectively towards long-term agendas of their own choosing.

In order to address the gap between momentary and sustained collaboration in computing research, we must also consider broader structural factors governing the production of academic knowledge. Klocker [48] describes the challenges of doing a $\mathrm{PhD}$ while also conducting participatory action research (PAR), a set of methods aligned with the community-based framework to which this project aspired. Participatory researchers are "not supposed to pre-empt how projects will unfold,' Klocker writes, and yet $\mathrm{PhD}$ students' degree progress may be impeded by community consultation. Despite this, Klocker takes a measured stance and suggests that building uncertainty into project proposals from the outset could ward against time crunches down the line. She also notes that tensions between individualism (doctoral work) and collectivism (participatory research) can arise during a $\mathrm{PhD}$, because the thesis is expected to be evidence of "individual research competence"; as such, "co-authorship is unacceptable" [48]. This tension can "reinforce unequal power relationships because $[\mathrm{PhDs}]$ are solely responsible for the task of putting collaborative research to paper... Concerns also exist over the rightful ownership of knowledge produced by a team." I faced this tension not just during the design process itself, but also while writing this paper.

Klocker points to the lack of "support and guidance" and the "limited availability of literature specific to PAR PhDs" in her field of geography research. I would argue that despite HCI and CSCW's strong leanings towards participatory and community-based work, more scaffolding in this area is needed for HCI scholars, especially doctoral students and early career researchers. This could take the form of articulating and reflecting on methods, challenges, failures, and opportunities for improvement in how we design with others. Following Harrington [40], such accounts must reckon with the social, political, and institutional contexts at play.

\subsection{Limitations}

The present account of our collaborative design process is limited by several factors. First of all, when Project Spectra was formed, I did not set out to study our design process. It was only upon reflection during the process that interesting and challenging "troubles" emerged. As mentioned early in the paper, I did not formally obtain consent from team members to include their own reflections here, for example via in-depth interviews. Future work could make "studying the process" central to the process itself, and include a more formal qualitative analysis of team members' experiences.

Second, because demographic information was not formally collected from each member of the group due to ethical obligations, it was impossible for this paper to provide a detailed characterization of the individuals who participated in Project Spectra. The "transgender community" is not a monolith, but this work flattens differences or dynamics related to race, gender, nationality, culture, language, and economic class. For example, all communication on our chat channels was in English, and we recruited members through English-speaking networks. Future studies of collaborative design should consider intra-community power and privilege dynamics as central to the analysis.

Third, the design process could have been more open, both within and outside our Discord channel. Design memo updates could have been posted directly to a public channel rather than to a Google Drive document. This might have fostered more group ownership of the design memo document, rather than it serving as a documentation space for the first author alone. Discord, despite its advantages, may have presented challenges to group members seeking to make sense of conversations they have missed, even though all messages were accessible and searchable (as is the case with other team communication channels [74]).

This work was also limited by the first author's academic environment, which, in some ways, also benefited the project. With my research stipend and university affiliation, I was able to work 
on Project Spectra full-time and had access to academic resources and collaborators. But these ostensible benefits also positioned me as a decision-maker and leader. Additional institutional training for working with (and within) community-based teams - as highlighted by Asad [6] would have better prepared me to conduct this work. As described in section 4.9, the fact that this work was conducted as part of an institutionally-mandated deliverable (my dissertation) had a significant effect on the design process. Future work could consider this issue at the outset, and plan around academic timelines and constraints to the fullest possible extent.

\subsection{Transformations}

This paper attempted to show how academic research contexts can reinforce power differentials and hamstring community collaborations. But can these problems be overcome, and if so, how? Mountz and colleagues argue for "slow scholarship," and "a fundamental restructuring of the university as a workplace and learning environment" [56]. The restructured academy would value care work, labor organizing and activism, collaborations, and collective authorship/ownership. Slow scholarship "raises the question of what counts and for whom and expands our community of care beyond those in the academy." How would the theory and practice of "community-based" design change in a restructured academy? How can we begin to live these values now?

Academic institutions often pride themselves on their contributions to local communities and economies, but these contributions are rooted in the exploitation of an expanding underclass of contingent workers (adjunct and non-tenure track faculty, who comprise upwards of 70 percent of the academic labor force [5]) and graduate student workers. Further, local activists have argued that the community contributions claimed by universities do more harm than good. Taking Northeastern University (my graduate institution) as an example, neighborhood developments and renovations have resulted in gentrification and a reduction in affordable housing availability $[19,65]$.

Examining at the underlying economic relationship between the university and the city reveals more. Although Northeastern University does not need to pay property taxes due to its non-profit status, the City of Boston requests payment in lieu of taxes (PILOT) totaling $\$ 11.4$ million. Of that sum, the university paid $\$ 1.7$ million and claimed an additional $\$ 5.7$ million in "community benefits," [65] including a nearby park renovation [53]. Effectively, the university undertakes gentrifying renovation projects - in which neighborhood residents do not have any meaningful democratic participation - to claim them as a tax write-off.

What does this have to do with community-based collaborative design? In the example above, the relationship between my university and local community is primarily extractive; Massachusetts State Representative Nika Elugardo, whose jurisdiction includes Northeastern's campus, said that "we need to shift the culture from a dominance model to a partnership model" [19]. Academic community-based work is necessarily influenced by this culture of dominance and extraction [40]; transforming it requires a massive shift, not only in institutional culture, but also in structure and governance.

As Alvarez argues [5], the academic labor force is essential to this transformation. Imagine a democratically-run university, with neighborhood councils, faculty unions, and student-run cooperatives collectively setting the priorities for teaching, research, and development. Deep, enduring ties to the surrounding community emerge organically from this arrangement, which would pave the way for collaborative research efforts that respond to the immediate needs and interests of the people. Online collaborative studies would also be improved this way. We envision a more interconnected and publicly accountable university, which would have more resources and opportunities for researchers to be trained and supported in doing community-based work thoughtfully and ethically. This is only one possible vision out of many, but nothing is possible unless we organize to change the status quo. 


\subsection{Process Recommendations}

Individual researchers can only act within imperfect systems; changing these systems will require collective action. My recommendations for researchers and practitioners interested in communitybased collaborative design are thus both individual and structural. I summarize them below, and point to locations within the paper where each point is elaborated on:

- Consider autoethnography as a method (via journaling/memoing) to reveal biases, contradictions, and emotional challenges arising within the design process. Encourage your collaborators to do the same. (Individual, see Section 4.1.)

- If considering online tools (such as Discord and FOSS), weigh the benefits and limitations they would bring to the process before deciding - as a group - if they are appropriate. Tools should be chosen on the basis of whether and how they would affect collective ownership and control of the project. (Individual, see Section 4.3.)

- Ask your institution to provide robust training and resources for conducting communitybased research, especially if you are in a department where such approaches are not commonly carried out. (Structural, see Section 5.3.)

- Organize with people both inside and outside the academy to address systemic marginalization. Expanding "our community of care beyond those in the academy" [56] requires recognizing the ways that the academy harms those outside it. For example, a researcher attempting to work with low-income communities surrounding their university should consider whether the university has policies of expansion that are displacing those same communities. (Structural, see Section 5.4.)

\section{CONCLUSION}

Community-based collaborative design allowed Project Spectra members to work against dominant, agency-stripping gender norms. Prioritizing agency in our app from the beginning, we supported users specifying and changing their goals at any time. Within our organization, we intended to support members' agency by fostering meaningful (and not mandatory) participation based on our own skills, interests, and backgrounds. We sought feedback from individuals outside our group, such as speech therapy practitioners, and recognized that they possessed unique training. However, we also did not elevate their knowledge above and beyond that of the broader community, especially if they hailed from a clinical institution. That being said, our work as a group was not immune to contradictions arising from my position in academia.

This paper attempted to answer calls within HCI for a "reorienting" and "restructuring" of PD, but more research on alternative working models is still needed. Researchers can use established groupwork models - such as PD, FOSS, and CBPP - as methodological guides to design; we can then ask how we can (and should) work with others, and what design methods are appropriate in a given context. Evaluative questions guided by feminist methodologies can reveal the relationships, habits, stories, and breakdowns that structure our design projects. This paper showed the importance of reflecting, via autoethnographic field notes, on the researcher's own positionality and influence on the research.

Coleman [18] describes how open source software developers act collectively but reject "politics," considering it a failed enterprise. Conversely, it seems, HCI researchers embrace political analyses and approaches but struggle to collectively challenge the oppressive systems that govern neoliberal academia. As a case in point, the academic context of this research, and my participation in it, sometimes benefited but ultimately hampered the extent to which our design process could embody a bottom-up and self-driven ideal. To address this issue, we must organize toward political, economic, and cultural shifts in the structure and governance of academic institutions. 


\section{ACKNOWLEDGMENTS}

The authors would like to thank all the members of Project Spectra. Additionally, Zheanna Erose and Lillith Whitmann both provided critical feedback and guidance to Project Spectra during our design process. Thanks to the Discord channels TransVoice and Scinguistics, and to all the anonymous individuals who completed our user tests. The first author would also like to thank Liz Polcha for sharing "slow scholarship" with me, and Halcyon Lawrence, Stephan Pennington, Barbara Worth, Michelle Borkin, and Meryl Alper for their kind support. This work was financially supported by the National Institutes of Health (Grant \#5F31DC016804-02) and the National Science Foundation’s Graduate Research Fellowship Program.

\section{REFERENCES}

[1] Richard K Adler, Sandy Hirsch, and Jack Pickering. 2018. Voice and communication therapy for the transgender/gender diverse client: A comprehensive clinical guide. Plural Publishing.

[2] Alex Ahmed, Bryan Kok, Coranna Howard, and Klew Still. 2020. Project Spectra: A Voice Training App for Transgender People. https://doi.org/10.5281/zenodo.3824662

[3] Alex A Ahmed. 2018. Trans Competent Interaction Design: A Qualitative Study on Voice, Identity, and Technology. Interacting with Computers 30, 1 (jan 2018), 53-71. https://doi.org/10.1093/iwc/iwx018

[4] Alex A Ahmed and Anna Lauren Hoffmann. 2018. Configuring the Trans Voice: Gender, Race, and Class in Mobile Voice Training Applications for Transgender People. In The 19th Annual Conference of the Association of Internet Researchers. Montreal, Canada.

[5] Maximillian Alvarez. 2017. Contingent No More: An academic manifesto. https:/thebaffler.com/the-poverty-oftheory/contingent-no-more The Baffler. Accessed 2020-08-25.

[6] Mariam Asad. 2019. Prefigurative design as a method for research justice. Proceedings of the ACM on Human-Computer Interaction 3, CSCW (2019). https://doi.org/10.1145/3359302

[7] Moya Bailey. 2015. \# transform (ing) DH Writing and Research: An Autoethnography of Digital Humanities and Feminist Ethics. DHQ: Digital Humanities Quarterly 9, 2 (2015).

[8] Liam Bannon, Jeffrey Bardzell, and Susanne Bødker. 2018. Introduction: Reimagining participatory design-Emerging voices. ACM Transactions on Computer-Human Interaction 25, 1 (2018), 1-8. https://doi.org/10.1145/3177794

[9] Eric P.S. Baumer and M. Six Silberman. 2011. When the implication is not to design (technology). Conference on Human Factors in Computing Systems - Proceedings (2011), 2271-2274. https://doi.org/10.1145/1978942.1979275

[10] Toby Beauchamp. 2019. Going stealth: Transgender politics and US surveillance practices. Duke University Press, 8-9.

[11] Diana Beirl, Anya Zeitlin, Jerald Chan, Kai Ip Alvin Loh, and Xiaodi Zhong. 2017. GotYourBack: An Internet of Toilets for the Trans* Community. In Proceedings of the 2017 CHI Conference Extended Abstracts on Human Factors in Computing Systems - CHI EA '17. ACM Press, New York, New York, USA, 39-45. https://doi.org/10.1145/3027063.3049272

[12] Ruha Benjamin. 2019. Race After Technology: Abolitionist Tools for the New fim Code. Polity Press, 217-239.

[13] Yochai Benkler and Helen Nissenbaum. 2006. Commons-based peer production and virtue. Journal of political philosophy 14, 4 (2006), 394-419.

[14] Cynthia L. Bennett and Daniela K. Rosner. 2019. The Promise of Empathy: Design, Disability, and Knowing the "Other" In Proceedings of the 2019 CHI Conference on Human Factors in Computing Systems (CHI '19). Association for Computing Machinery, New York, NY, USA, Article 298, 13 pages. https://doi.org/10.1145/3290605.3300528

[15] Talia Mae Bettcher. 2006. Appearance, Reality and Gender Deception: Reflections on Transphobic Violence and the Politics of Pretence. Violence, Victims, fustifications: Philosophical Approaches (2006), 175-200.

[16] Christian Bird, David Pattison, Raissa D’Souza, Vladimir Filkov, and Premkumar Devanbu. 2008. Latent social structure in open source projects. Proceedings of the ACM SIGSOFT Symposium on the Foundations of Software Engineering January (2008), 24-35. https://doi.org/10.1145/1453101.1453107

[17] Lisa Carew, Georgia Dacakis, and Jennifer Oates. 2007. The effectiveness of oral resonance therapy on the perception of femininity of voice in male-to-female transsexuals. fournal of Voice 21, 5 (2007), 591-603.

[18] Gabriella Coleman. 2009. Code is Speech: Legal Tinkering, Expertise, and Protest among Free and Open Source Software Developers. Cultural Anthropology 24, 3 (aug 2009), 420-454. https://doi.org/10.1111/j.1548-1360.2009.01036.x

[19] Sean Philip Cotter. 2020. Roxbury leaders: Northeastern has lost its way. https://www.bostonherald.com/2020/01/12 roxbury-leaders-northeastern-has-lost-its-way/ The Boston Herald. Accessed 2020-08-25.

[20] Georgia Dacakis, Jennifer Oates, and Jacinta Douglas. 2012. Beyond voice: Perceptions of gender in male-to-female transsexuals. Current opinion in otolaryngology \& head and neck surgery 20, 3 (2012), 165-170.

[21] Shelagh Davies, Vikt Oria, Viktória G Papp, and Christella Antoni. 2015. Voice and Communication Change for Gender Nonconforming Individuals: Giving Voice to the Person Inside. International fournal of Transgenderism 16, 3 (2015),

Proc. ACM Hum.-Comput. Interact., Vol. 4, No. CSCW3, Article 258. Publication date: December 2020. 
117-159. https://doi.org/10.1080/15532739.2015.1075931

[22] P Descloux, S Isoard-Nectoux, B Matoso, L Matthieu-Bourdeau, F Schneider, and V Schweizer. 2012. Transsexuality: speech therapy supporting the" voice" of transformation. Revue de laryngologie-otologie-rhinologie 133, 1 (2012), 41-44.

[23] Nicolas Ducheneaut. 2005. Socialization in an open source software community: A socio-technical analysis. Computer Supported Cooperative Work: CSCW: An International fournal 14, 4 (2005), 323-368. https://doi.org/10.1007/s10606005-9000-1

[24] Florian Echtler and Maximilian Häußler. 2018. Open source, open science, and the replication crisis in HCI. Conference on Human Factors in Computing Systems - Proceedings 2018-April (2018), 1-8. https://doi.org/10.1145/3170427.3188395

[25] E. Kale Edmiston, Cameron A. Donald, Alice Rose Sattler, J. Klint Peebles, Jesse M. Ehrenfeld, and Kristen Laurel Eckstrand. 2016. Opportunities and Gaps in Primary Care Preventative Health Services for Transgender Patients: A Systematic Review. Transgender Health 1, 1 (2016), 216-230. https://doi.org/10.1089/trgh.2016.0019

[26] Coraline Ada Ehmke. 2014. Contributor Covenant. https://www.contributor-covenant.org/version/1/4/code-ofconduct

[27] Carolyn Ellis, Tony E Adams, and Arthur P Bochner. 2010. Autoethnography: An Overview. Forum Qualitative Sozialforschung / Forum: Qualitative Social Research 12, 1 (2010), 273-290. https://doi.org/10.17169/fqs-12.1.1589

[28] Robert M Emerson, Rachel I Fretz, and Linda L Shaw. 2011. Writing ethnographic fieldnotes. University of Chicago Press, 123-127.

[29] Marylou Pausewang Gelfer and Bethany Ramsey Van Dong. 2013. A preliminary study on the use of vocal function exercises to improve voice in male-to-female transgender clients. Fournal of Voice 27, 3 (2013), 321-334.

[30] Danielle M. Giffort and Kelly Underman. 2016. The relationship between medical education and trans health disparities: a call to research. Sociology Compass 10, 11 (2016), 999-1013. https://doi.org/10.1111/soc4.12432

[31] Jaime M. Grant, Lisa A. Mottet, Justin Tanis, Jack Harrison, Jody L Herman, and Mara Keisling. 2011. Injustice at Every Turn: A Report of the National Transgender Discrimination Survey. Technical Report. https://doi.org/10.1016/S00167878(90)80026-2

[32] Collin Green, Alex Eiser, Irene Tollinger, Lanie Castro, Christian Ratterman, Alonso Vera, and Guy Pyrzak. 2009. Leveraging open-source software in the design and development process. Conference on Human Factors in Computing Systems - Proceedings (2009), 3061-3074. https://doi.org/10.1145/1520340.1520433

[33] Critical Platform Studies Group, Lilly Irani, Niloufar Salehi, Joyojeet Pal, Andrés Monroy-Hernández, Elizabeth Churchill, and Sneha Narayan. 2019. Patron or Poison?. In Conference Companion Publication of the 2019 on Computer Supported Cooperative Work and Social Computing - CSCW'19. ACM Press, New York, New York, USA, 111-115. https://doi.org/10.1145/3311957.3358610

[34] Oliver Haimson. 2018. Social Media as Social Transition Machinery. Proc. ACM Hum.-Comput. Interact. 2, CSCW, Article 63 (Nov. 2018), 21 pages. https://doi.org/10.1145/3274332

[35] Oliver L Haimson, Jed R Brubaker, Lynn Dombrowski, and Gillian R Hayes. 2016. Digital Footprints and Changing Networks During Online Identity Transitions. In Proceedings of the 2016 CHI Conference on Human Factors in Computing Systems - CHI '16. ACM Press, New York, New York, USA, 2895-2907. https://doi.org/10.1145/2858036.2858136

[36] Oliver L. Haimson, Dykee Gorrell, Denny L. Starks, and Zu Weinger. 2020. Designing Trans Technology: Defining Challenges and Envisioning Community-Centered Solutions. Conference on Human Factors in Computing Systems Proceedings (2020), 1-13.

[37] Donna J Haraway. 2016. Staying with the trouble: Making kin in the Chthulucene. Duke University Press.

[38] Teresa LD Hardy, Carol A Boliek, Kristopher Wells, and Jana M Rieger. 2013. The ICF and male-to-female transsexual communication. International fournal of Transgenderism 14, 4 (2013), 196-208.

[39] Christina N. Harrington, Katya Borgos-Rodriguez, and Anne Marie Piper. 2019. Engaging Low-Income African American Older Adults in Health Discussions through Community-based Design Workshops. Proceedings of the 2019 ACM Conference on Human Factors in Computing Systems (2019), 1-15. https://doi.org/10.1145/3290605.3300823

[40] Christina N. Harrington, Sheena Erete, and Anne Marie Piper. 2019. Deconstructing community-based collaborative design: Towards more equitable participatory design engagements. Proceedings of the ACM on Human-Computer Interaction 3, CSCW (2019). https://doi.org/10.1145/3359318

[41] Lilly Irani. 2018. "Design Thinking”: Defending Silicon Valley at the Apex of Global Labor Hierarchies. Catalyst: Feminism, Theory, Technoscience 4, 1 (2018), 1-19. https://doi.org/10.28968/cftt.v4i1.29638

[42] Lilly Irani. 2019. Chasing Innovation: Making Entrepreneurial Citizens in Modern India. Princeton University Press.

[43] Lilly Irani, Janet Vertesi, Paul Dourish, Kavita Philip, and Rebecca E. Grinter. 2010. Postcolonial Computing: A Lens on Design and Development. Proceedings of the 28th international conference on Human factors in computing systems - CHI '10 (2010), 1311. https://doi.org/10.1145/1753326.1753522

[44] Sandy E. James, Jody L. Herman, Susan Rankin, Mara Keisling, Lisa Mottet, and Ma'ayan Anafi. 2016. The Report of the 2015 US Transgender Survey. Technical Report. 
[45] Os Keyes. 2018. The misgendering machines: Trans/HCI implications of automatic gender recognition. Proceedings of the ACM on Human-Computer Interaction 2, CSCW (2018). https://doi.org/10.1145/3274357

[46] Os Keyes, Josephine Hoy, and Margaret Drouhard. 2019. Human-Computer Insurrection: Notes on an Anarchist HCI. In Proceedings of the 2019 CHI Conference on Human Factors in Computing Systems (CHI '19). Association for Computing Machinery, New York, NY, USA, Article 339, 13 pages. https://doi.org/10.1145/3290605.3300569

[47] Vera Khovanskaya and Phoebe Sengers. 2019. Data Rhetoric and Uneasy Alliances: Data Advocacy in US Labor History. Proceedings of the 2019 on Designing Interactive Systems Conference - DIS '19 (2019), 1391-1403. https: //doi.org/10.1145/3322276.3323691

[48] Natascha Klocker. 2012. Doing Participatory action research and Doing a PhD: Words of encouragement for prospective students. fournal of Geography in Higher Education 36, 1 (2012), 149-163. https://doi.org/10.1080/03098265.2011.589828

[49] Sara LeGrand, Kathryn Elizabeth Muessig, Tobias McNulty, Karina Soni, Kelly Knudtson, Alex Lemann, Nkechinyere Nwoko, and Lisa B Hightow-Weidman. 2016. Epic Allies: Development of a Gaming App to Improve Antiretroviral Therapy Adherence Among Young HIV-Positive Men Who Have Sex With Men. FMIR Serious Games 4, 1 (2016), e6. https://doi.org/10.2196/games.5687

[50] Sara LeGrand, Kathryn E. Muessig, Alyssa Platt, Karina Soni, Joseph R. Egger, Nkechinyere Nwoko, Tobias McNulty, and Lisa B. Hightow-Weidman. 2018. Epic allies, a gamified mobile phone app to improve engagement in care, antiretroviral uptake, and adherence among young men who have sex with men and young transgender women who have sex with men: Protocol for a randomized controlled trial. fournal of Medical Internet Research 20, 4 (2018) https://doi.org/10.2196/resprot.8811

[51] VoxPop LLC. 2020. EVA: Transgender Voice App. http://exceptionalvoiceapp.com/

[52] Speechtools Ltd. 2020. Christella VoiceUp. https://speechtools.co/christella-voiceup

[53] Greg St. Martin. 2018. Northeastern to open state-of-the-art community park in Boston. https://news.northeastern. edu/2018/08/02/northeastern-prepares-to-open-state-of-the-art-community-park-in-boston/ News Northeastern. Accessed 2020-08-25.

[54] Christopher Mendez, Margaret Burnett, Hema Susmita Padala, Zoe Steine-Hanson, Claudia Hilderbrand, Amber Horvath, Charles Hill, Logan Simpson, Nupoor Patil, and Anita Sarma. 2018. Open source barriers to entry, revisited. (2018), 1004-1015. https://doi.org/10.1145/3180155.3180241

[55] Eunyoung Moon. 2016. Do open projects 'break the mirror'?: Re-conceptualization of organizational configurations in Free/Libre Open Source Software (FLOSS) development. Proceedings - 11th IEEE International Conference on Global Software Engineering Companion Proceedings, ICGSEW 2016 (2016), 73-76. https://doi.org/10.1109/ICGSEW.2016.18

[56] Alison Mountz, Anne Bonds, Becky Mansfield, Jenna Loyd, Jennifer Hyndman, Margaret Walton-Roberts, Ranu Basu, Risa Whitson, Roberta Hawkins, Trina Hamilton, and Winifred Curran. 2015. For slow scholarship: A feminist politics of resistance through collective action in the Neoliberal University. Acme 14, 4 (2015), 1235-1259.

[57] Joyojeet Pal. 2017. CHI4Good or Good4CHI. Proceedings of the 2017 CHI Conference Extended Abstracts on Human Factors in Computing Systems - CHI EA '17 (2017), 709-721. https://doi.org/10.1145/3027063.3052766

[58] Stephan Pennington. 2019. Transgender Passing Guides and the Vocal Performance of Gender and Sexuality. In The Oxford Handbook of Music and Queerness. 1-44. https://doi.org/10.1093/oxfordhb/9780199793525.013.65

[59] Guilherme C. Pereira and M. Cecilia C Baranauskas. 2010. Codesigning emancipatory systems: a study on mobile applications and lesbian, gay, bisexual, and transgender (LGBT) issues. SBC fournal on Interactive Systems 9, 3 (2010), 80-92. https://www.seer.ufrgs.br/jis/article/view/80234

[60] Tonia Poteat, Danielle German, and Colin Flynn. 2016. The conflation of gender and sex: gaps and opportunities in HIV data among transgender women and MSM. Global public health 11, 7-8 (2016), 835-848.

[61] Purr Programming. 2020. Voice Pitch Analyzer. https://play.google.com/store/apps/details?id=de.lilithwittmann. voicepitchanalyzer\&hl=en

[62] Daniela K Rosner. 2018. Critical Fabulations: Reworking the Methods and Margins of Design. MIT Press.

[63] Morgan Klaus Scheuerman, Stacy M. Branham, and Foad Hamidi. 2018. Safe spaces and safe places: Unpacking technology-mediated experiences of safety and harm with transgender people. Proceedings of the ACM on HumanComputer Interaction 2, CSCW (2018). https://doi.org/10.1145/3274424

[64] Julia M. Serano. 2008. A matter of perspective: A transsexual woman-centric critique of Dreger's "scholarly history" of the Bailey controversy. Archives of Sexual Behavior 37, 3 (2008), 491-494. https://doi.org/10.1007/s10508-008-9332-2

[65] Boston Herald Editorial Staff. 2020. Northeastern should stop shorting Roxbury. https://www.bostonherald.com/2020/ 01/14/northeastern-should-stop-shorting-roxbury/ The Boston Herald. Accessed 2020-08-25

[66] Denny L. Starks, Tawanna Dillahunt, and Oliver L. Haimson. 2019. Designing technology to support safety for transgender women \& non-binary people of color. DIS 2019 Companion - Companion Publication of the 2019 ACM Designing Interactive Systems Conference (2019), 289-294. https://doi.org/10.1145/3301019.3323898

[67] Angelika Strohmayer, Jenn Clamen, and Mary Laing. 2019. Technologies for social justice lessons from sex workers on the front lines. Conference on Human Factors in Computing Systems - Proceedings (2019), 1-14. https://doi.org/10.1145/ 
3290605.3300882

[68] Project Spectra Team. [n.d.]. Project Spectra. https://github.com/project-spectra/ Github Organization. Accessed 2020-04-13.

[69] Tourmaline, Eric A. Stanley, and Johanna Burton. 2017. Known Unknowns: An Introduction to Trap Door. In Trap Door: Trans Cultural Production and the Politics of Visibility, Tourmaline, Eric A. Stanley, and Johanna Burton (Eds.) MIT Press/the New Museum, Cambridge, MA/New York, NY, xv-xxiv.

[70] Evan Vipond. 2015. Resisting Transnormativity: challenging the medicalization and regulation of trans bodies. Theory in Action 8, 2 (2015), 21-44. https://doi.org/10.3798/tia.1937-0237.15008

[71] Aimee Wodda and Vanessa Panfil. 2015. "Don't Talk to Me about Deception": The Necessary Erosion of the Trans-Panic Defense. Albany Law Review 78, 3 (2015), 927.

[72] Virginia I Wolfe, David L Ratusnik, Furman H Smith, and Gretajo Northrop. 1990. Intonation and fundamental frequency in male-to-female transsexuals. Journal of Speech and Hearing Disorders 55, 1 (1990), 43-50.

[73] Zegaye Seifu Wubishet, Bendik Bygstad, and Prodromos Tsiavos. 2013. A Participation Paradox: Seeking the Missing Link between Free/Open Source Software and Participatory Design. fournal of Advances in Information Technology 4 , 4 (nov 2013), 181-193. https://doi.org/10.4304/jait.4.4.181-193

[74] Amy X. Zhang and Justin Cranshaw. 2018. Making sense of group chat through collaborative tagging and summarization. Proceedings of the ACM on Human-Computer Interaction 2, CSCW (2018). https://doi.org/10.1145/3274465

Received January 2020; revised June 2020; accepted July 2020 